\title{
Der Migrationshintergrund als Grenze der Palliativversorgung am Lebensende?
}

\section{Can migration background be a boundary in palliative care at the end of life?}

\author{
Autoren \\ Christian Banse ${ }^{1}$, Sonja Owusu-Boakye ${ }^{1,2}$, Franziska Schade ${ }^{1}$, Maximiliane Jansky¹, Gabriella Marx ${ }^{1,3}$, Friedemann Nauck ${ }^{1}$
}

\section{(c) (1) $\circledast \oplus$}

Institute

1 Klinik für Palliativmedizin, Universitätsmedizin Göttingen

2 Department Pflege und Management, Hochschule für Angewandte Wissenschaften Hamburg

3 Institut und Poliklinik für Allgemeinmedizin, Universitätsklinikum Hamburg-Eppendorf

\section{Schlüsselwörter}

Menschen mit Migrationshintergrund in Deutschland, Palliativversorgung, Krebserkrankung, qualitative Studie, Versorgungserfahrungen

Key words

individuals in Germany with a migration background, palliative care, cancer, qualitative study, care experiences

online publiziert 17.11 .2020

Bibliografie

Dtsch Med Wochenschr 2020; 145: e22-e28

DOI 10.1055/a-1263-3437

ISSN 0012-0472

(c) 2020. The Author(s).

This is an open access article published by Thieme under the terms of the Creative Commons Attribution-NonDerivative-NonCommercial License, permitting copying and reproduction so long as the original work is given appropriate credit. Contents may not be used for commecial purposes, or adapted, remixed, transformed or built upon. (https://creativecommons.org/licenses/by-nc-nd/4.0/)

Georg Thieme Verlag KG, Rüdigerstraße 14,

70469 Stuttgart, Germany

Korrespondenzadresse

Dr. Christian Banse

Klinik für Palliativmedizin, Universitätsmedizin Göttingen, Von-Siebold-Straße 3, 37075 Göttingen, Deutschland Tel.: +49/5 51/3960555

Christian.Banse@med.uni-goettingen.de

\section{ZUSAMMENFASSUNG}

Hintergrund Menschen mit Migrationshintergrund nutzen nach dem Stand der Forschung das palliative Versorgungsangebot wenig. Die möglichen Gründe sind weitgehend unklar. Häufig werden kulturelle Unterschiede geltend gemacht. Eine Untersuchung der Bedürfnisse von krebskranken Patienten mit Migrationshintergrund, ihren Angehörigen und medizinischen und pflegenden Versorgenden in Deutschland bietet einen Überblick über die Versorgungsprobleme.

Methoden In dem qualitativen Forschungsdesign stehen biografische Erzählungen der Patienten, ihrer Angehörigen und der medizinischen und pflegenden Versorgenden im Vordergrund. Mit der Grounded Theory wurde eine Zeile-für-ZeileAnalyse der Daten durchgeführt, mit der verschiedene Kategorien und Handlungsstrategien im Umgang mit der Krankheit und der Versorgungssituation gefunden werden konnten.

Ergebnisse Wenn schwerkranke Patienten mit Migrationshintergrund in einer palliativen Versorgungsstruktur ankommen, erleben sie in dieser politische und soziale Abgrenzungen. Migrationsspezifische Einflüsse erschweren deshalb die Bearbeitung der Krankheit und die palliativmedizinische Behandlung und Versorgung am Lebensende. Angehörige und medizinische und pflegende Versorgende erleben die Situation als eine Herausforderung, weil sie den Erwartungen der Patienten nicht entsprechen können und auf Übersetzer und Vermittler angewiesen sind. Viele Probleme werden kulturellen Unterschieden zugerechnet, obwohl die Patienten am Lebensende eher allgemeine Bedürfnisse formulieren.

Schlussfolgerung Die Palliativversorgung von Menschen mit Migrationshintergrund findet häufig ohne spezifische Kenntnisse der Migrationsbiografie statt. Um den politischen und sozialen Einflüssen gerecht zu werden, bedarf es einer Unterstützung, die die Migrationsbiografie berücksichtigt.

\section{ABSTRACT}

Background According to current research data, individuals with migration background rarely utilize palliative care services. The potential reasons remain largely unclear, with cultural differences being suggested most frequently. A study focusing on the needs of cancer patients with migration background in Germany provides an overview of the care problems encountered. Method The qualitative study design focuses on biographic narratives by patients, their relatives and medical care providers $(n=57)$. Data were analyzed with "grounded theory" which disclosed various categories and coping strategies in dealing with both the disease and the care provided.

Result Arriving in palliative care, severely ill patients with migration background experience social exclusion. These migration- 
specific effects have a negative impact on coping with the disease and on palliative treatment and end-of-life care. Relatives and medical care providers face a challenge when they fail to meet the patients' expectations. Many problems are attributed to cultural differences although patients nearing the end of their lifes tend to articulate rather generic needs.
Conclusion Individuals with migration background frequently receive palliative care without their care givers having any specific knowledge of their migration biography. In order to do justice to societal effects, the support given must go beyond the mere medical expertise.

\section{Einleitung}

\section{Der Migrationshintergrund als gesundheitspolitische Herausforderung}

Menschen, die nach Deutschland migriert sind, stehen zunehmend im Fokus öffentlicher Diskurse, in denen grundlegende Fragen zu ihrer Integration in die Gesellschaft aufgeworfen werden [1]. So wird im Zuge des vergleichsweise hohen Aufkommens von Geflüchteten in den Jahren 2015/2016 angenommen, dass das Gesundheitssystem vor neuen Herausforderungen steht [2], gerade was die Öffnung für alle gesellschaftlichen Gruppen betrifft. Dieser Einschätzung liegt die Annahme zugrunde, dass es für Asylsuchende keinen chancengleichen Zugang zum Gesundheitssystem gibt [3]. Auch Menschen, die über einen gesicherten Aufenthaltsstatus verfügen, können im Vergleich zur Mehrheitsgesellschaft einer kumulativen Benachteiligung durch Arbeitsbedingungen, Wohnumfeld, migrationsbedingte Familientrennung und Diskriminierungen unterliegen, die sich auf die Integration in das Gesundheitssystem auswirkt [4-10].

Erst seit wenigen Jahren wird in Deutschland zur Palliativversorgung von Menschen mit Zuwanderungsgeschichte geforscht und eine geringe Inanspruchnahme des Angebots vermutet [11]. Die Studien befassen sich jedoch entweder lediglich mit je einer Gruppe mit spezifischem Herkunftskontext [12, 13] oder fokussieren ausschließlich die Perspektive von Versorgenden aus dem Gesundheitssystem [14] oder die der Patienten [9]. In verschiedenen Veröffentlichungen werden kulturelle Aspekte als Grund für einen unterschiedlichen Umgang mit dem Angebot diskutiert [15-18]. Doch fehlen empirisch fundierte Reflexionen dazu, ob tatsächlich „Kultur“ für Unterschiede in der Versorgung verantwortlich ist oder nicht eher Migrationserfahrungen, der Herkunftskontext oder die Zuordnung zu einer bestimmten Gruppierung relevant werden.

Der vorliegende Beitrag basiert auf den Ergebnissen einer von der Deutschen Krebshilfe geförderten Studie (Fördernummer 111579), in der Palliativpatienten mit Migrationshintergrund und einer fortgeschrittenen Krebserkrankung, deren Angehörige sowie medizinisch und pflegend Tätige zur Versorgungssituation befragt wurden. Laut Statistischem Bundesamt hat eine Person einen Migrationshintergrund, „wenn sie selbst oder mindestens ein Elternteil die deutsche Staatsangehörigkeit nicht durch Geburt besitzt“ [19]. Ziel war es, die Bedürfnisse der Patienten aus deren Perspektive zu erfahren und nach Barrieren für eine diesen Bedürfnissen angemessene Versorgung zu suchen. Insbesondere die Bedeutung der durch die Migration geprägten Biografie für die Versorgungssituation stand im Vordergrund, weil so Verallgemeinerungen über diese heterogene Gruppe der Patienten vermieden und zugleich Migration als ein auf vielfältige Weise das Leben bestimmendes Phänomen in den Blick genommen werden konnten. Forschungsleitend war die Frage, ob es migrationsspezifische Einflüsse gibt, die die Versorgungssituation der an Krebs erkrankten Palliativpatienten negativ beeinflussen. Die Interviews mit Angehörigen und Versorgenden sowie Schnittstellenakteuren ermöglichten darüber hinaus, unterschiedliche Perspektiven zu berücksichtigen.

\section{Methode}

Der offenen Forschungsfrage entsprach das qualitativ-methodische Vorgehen, alle Interviews mit einem narrativen Gesprächsansatz zu führen [20]. Die Interviews wurden mit dem interpretativen Verfahren der Grounded Theory in einer Zeile-für-ZeileAnalyse ausgewertet, um auch ihren latenten Sinn herauszuarbeiten [21]. Indem die Aussagen fallimmanent und fallübergreifend verglichen wurden, konnte für jede Gruppe (Patienten, Angehörige, medizinische und pflegende Versorgende) ein zentrales Phänomen herausgearbeitet werden.

Mit der Grounded Theory wurde eine soziologische Theorieperspektive gewählt, die Realität als von den Handelnden hervorgebracht versteht [22]. Es geht darum, wie die Interviewten Situationen in ihrer jeweiligen Perspektive deuten. Mittels der interpretativen Analyse werden Einblicke in soziale Zusammenhänge möglich, die über die medizinischen hinausgehen.

\section{Studienteilnehmer}

Im Zeitraum 10/2015-08/2017 wurden 57 Personen in 5 deutschen Städten interviewt ( $\triangleright$ Tab. 1). Alle Studienteilnehmer wurden in ihrer Muttersprache über den Zweck und die Durchführung der Studie aufgeklärt. Nach Bedarf wurden die Interviews mit Sprachmittlern durchgeführt. Die Daten wurden wörtlich transkribiert und gemäß den Richtlinien der Ethikkommission der Universitätsmedizin Göttingen pseudonymisiert und sicher aufbewahrt (Ethikvotum Nr. 24/3/15). Weitere Ethikvoten wurden von den zuständigen lokalen Ethikkommissionen eingeholt. Der Auswertungsprozess erfolgte intersubjektiv, sodass die Kategorien ständig im Forscherteam überprüft wurden.

\section{Ergebnisse}

Die Struktur der Ergebnisdarstellung folgt den jeweils zentralen Kategorien der unterschiedlichen Erhebungsgruppen. Die folgenden Aussagen sind aus Gründen der Lesbarkeit geglättet. 
- Tab. 1 An der Studie teilgenommene Gruppen.

Patienten $(n=21)$

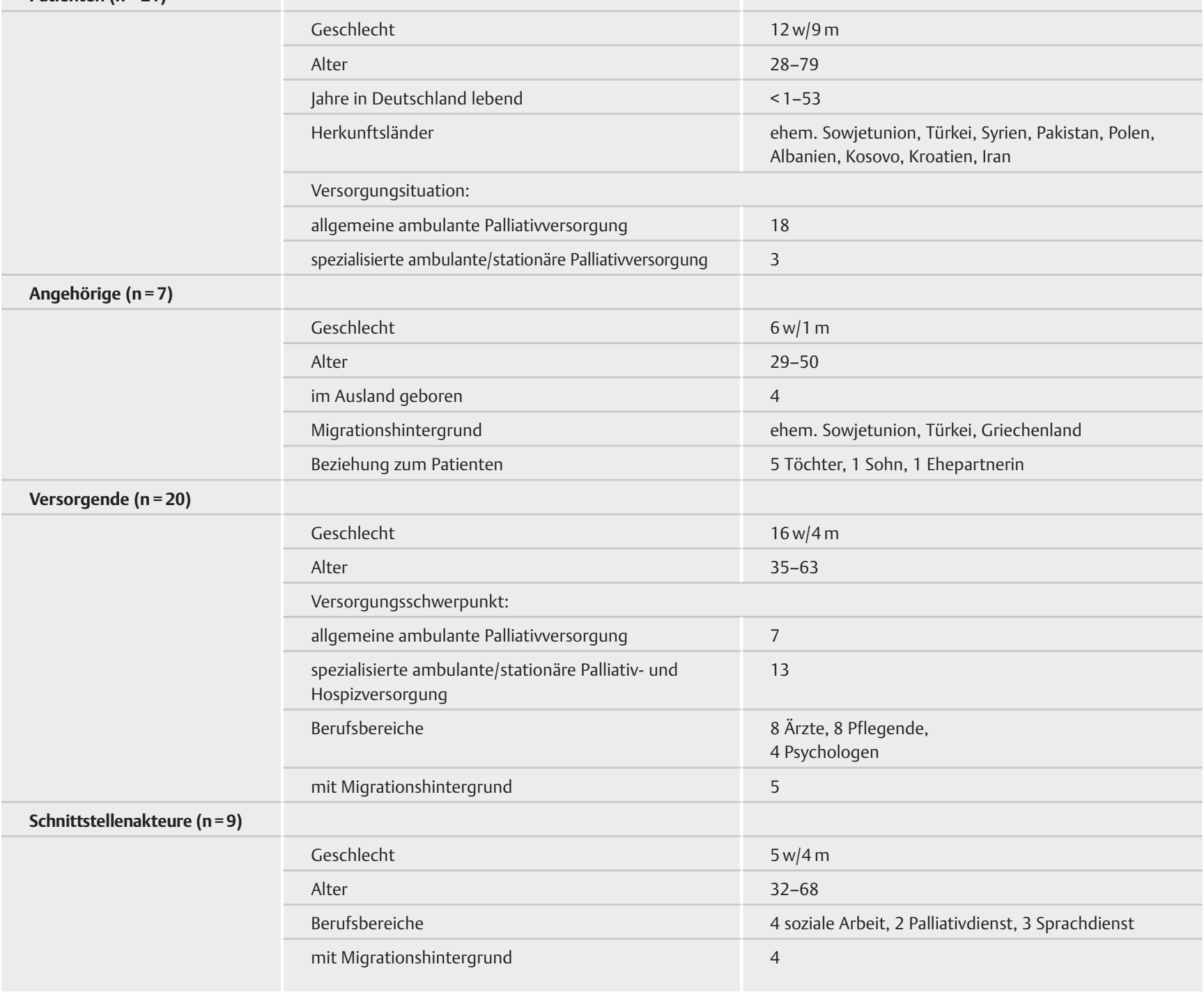

\section{Patienten: Migration und Krankheit als Faktoren für die Erfahrung von Ausgrenzung}

Als ein Ergebnis der Studie kann festgehalten werden, dass von den schwerkranken Patienten mit Migrationshintergrund Bedürfnisse für ihre Versorgungssituation formuliert werden, die nicht migrationsspezifisch sind: Patienten wünschen sich von den Versorgenden neben medizinische auch soziale Kompetenzen. Gleichzeitig erleben sie bürokratische Hindernisse und soziale Abgrenzungen. Viele Patienten haben das Gefühl, im Gesundheitssystem insgesamt wegen ihrer Herkunft anders behandelt zu werden als Menschen ohne Migrationshintergrund (Kasten 1, Zitat 1).

Bei Sprachgrenzen sind Patienten darauf angewiesen, dass eine funktionierende Kommunikationsstruktur aus Dolmetschern, Familienangehörigen und Unterstützungsnetzwerken vorhanden ist.

Patienten meinen, dass sich die Versorger zu wenig Zeit für sie nehmen. Deshalb sind die von den Patienten am meisten geschätzten Versorger diejenigen, die sich für ihre Patienten nicht nur medizinisch engagieren, sondern auch bei sozialen und rechtlichen Fragen aktiv werden.

Mit der Migration nach Deutschland erleben die Patienten einen ökonomischen und sozialen Statusverlust, der durch den Befund einer Krebserkrankung verstärkt wird und bei den Interviewten das Bedürfnis hervorruft, ihre Situation zu rechtfertigen. Die mit Migration und Krankheit einhergehende Scham, besonders dem Umfeld und z. T. auch dem medizinischen Personal gegenüber, zeigt sich in den Interviews dadurch, dass die Normalität bisheriger Rollenkonzepte aufrechterhalten werden soll. Dieser Anspruch steht im Konflikt mit der Krankheitsrealität, die zunehmend mehr Raum im Lebensalltag der Patienten einnimmt. Bei Müttern mit jüngeren Kindern kann das Problem relevant sein, genderspezifische Rolleneigenschaften in der Familie erfüllen zu wollen, während zugleich der Wunsch nach Entlastung bei der Kinderbetreuung besteht. In einigen Interviews wird deutlich, dass die Patienten in den Familien (oder auch Freunden und Bekannten gegenüber) nicht offen kommunizieren, was sie bewegt und was sie sich wünschen. Viele betonen, dass sie ihren Familien nicht 
zur Last fallen wollen (Kasten 1, Zitat 2). Das deutsche Gesundheitssystem wird von einigen Menschen mit Migrationshintergrund als letzte Chance gesehen, eine adäquate medizinische Versorgung zu erhalten, da diese im Herkunftsland aufgrund von medizinischen Standards, Krisen oder Krieg nicht (mehr) gewährleistet werden kann (Kasten 1, Zitat 3). Einige Menschen haben im weit fortgeschrittenen Krankheitsstadium eine Migration auf sich genommen und ihr Vermögen auf der Suche nach einer besseren medizinischen Versorgung ausgegeben. Auch hier zeigen sich migrationsspezifische Einflüsse auf die Versorgung, denn mehrere interviewte Patienten sind von ihren Kindern oder anderen Familienangehörigen getrennt, da diese kein Visum bekommen oder aus finanziellen Gründen die Reise nicht antreten können. Patienten artikulieren Schuldgefühle und formulieren vermeintliche oder tatsächliche Erwartungen der Familie als eigene Ansprüche, da man sich Geld von Verwandten für eine Versorgung geliehen hat, von der man sich Heilung verspricht.

Viele Patienten haben eine oft jahrelange therapeutische und diagnostische Odyssee hinter sich, bis es zum Befund gekommen ist. Die unerwartete Diagnose dann in Deutschland geht bei einigen Patienten mit dem Gefühl von ungerechter Behandlung einher. Man glaubt zunächst, die Diagnosestellung ist ein Ausdruck von Diskriminierung. Das medizinische System erleben sie als ein Netz aus unterschiedlichen medizinischen Kompetenzen, das für manche Patienten unübersichtlich bleibt. Sie nehmen Auseinandersetzungen an den Schnittstellen zwischen den onkologischen Stationen und der Palliativmedizin wahr und fühlen sich zu wenig informiert.

\section{KASTEN 1: AUSSAGEN AUS DEN WÖRTLICH TRANSKRIBIERTEN PATIENTENINTERVIEWS}

Zitat 1: „viel Information kriegt man nicht so muss man damit leben eigentlich, als Ausländerin wenn man Krankenhaus kommt und so weiter, ist schlimm, ganz ganz schlimm, erstmal Mentalität und zweitens wenn man die Sprache nicht versteht bist du halt dümmer gestellt, man ist ja automatisch anders behandelt"

Frau B. ${ }^{1}$, Türkei, 69J.

Zitat 2: „es ist sehr schwer gefallen, weil die Krankheit dauert fast drei Jahre, und leider, was ich vorher alles versucht habe, hatte so viel gekostet dass was alles wir gespart haben mussten alles geben, und nicht nur wir sondern, auch, die, Mitglied von Familien, was bedeutet dass sie sind auch in einen, Zustand gekommen das, die nicht mehr, helfen können aber, auch sind sie nicht in der Lage, hier, mich zu besuchen zu kommen weil es kostet das Geld und die schaffen das nicht" Herr T., Albanien, 45J.

Zitat 3: „ich startete die Chemo in Syrien, aber es war sehr schwierig für mich, weil es ist so teuer, schwer eine Chemotherapie zu finden weil ich kaufte auf dem Schwarzmarkt, weil wir keine Medikamente in den Krankenhäusern finden konnten keine, keine Elektrizität, keine Doktoren und, nein das ist die Situation, die medizinische Situation in den Krankenhäusern in Syrien.“ Frau M., Syrien, $51 \mathrm{~J}$.

1

\section{Angehörige: Als Anwalt der Patienten - Das „Richtige“ tun wollen}

Angehörige haben eine spezifische Perspektive beim Blick auf die Behandlungssituation. Sie stehen oft als Sprach- und Interessenvermittler zwischen den Patienten und dem medizinischen Personal. Das Verhältnis zum kranken Verwandten wird dadurch neu definiert. Angehörige berichten von dem Gefühl der Hilflosigkeit und Handlungsohnmacht der Patienten, wenn diese Schwierigkeiten haben, sich sprachlich zu verständigen, ein direkter Austausch über die Diagnose oder Prognose der Erkrankung durch den Patienten vermieden wird oder Hemmungen bestehen, über Schwierigkeiten in der Versorgung zu sprechen. Aus diesen Situationen entsteht das Gefühl der Verantwortung dem Patienten gegenüber. Der Anspruch von Kindern, den Eltern eine bestmögliche Versorgung zu garantieren, ist eine Haltung, die vor allem von den Mitgliedern der 2. Generation der migrierten Familien eingenommen wird, weil ihnen von den erkrankten Familienmitgliedern ein besseres Leben in Deutschland ermöglicht wurde. Sie verstehen sich als ,Anwalt' ihrer kranken Verwandten, die diesen zu ihrem Recht auf angemessene Versorgung verhelfen (Kasten 2, Zitat 4).

Diese Ansprüche der Angehörigen können zu inneren Konflikten führen, wenn Angehörige eine ähnliche Handlungsohnmacht im Gesundheitssystem erleben wie ihre erkrankten Verwandten und der Erwartungsdruck zu hoch wird, für die anfallenden Probleme in der Versorgung eine Lösung zu finden, die den Bedürfnissen des Patienten entspricht.

Als besonders belastend wird die Position empfunden, die den Ärzten gegenüber entsteht: In ihrer Rolle als Sprachmittler, auf die zuweilen spontan zurückgegriffen wird, sehen sich die Angehörigen mit den medizinischen Untersuchungsergebnissen in einer Unmittelbarkeit konfrontiert, die wenig Raum für eine eigene Verarbeitung der Krankheitssituation lässt (Kasten 2, Zitat 5). Aus Sicht der Angehörigen wird von Ärzten wenig bedacht, dass diese den Patienten vor allem als Angehörige und nicht als Übersetzer begleiten. Obwohl sie einen Bedarf an Unterstützung formulieren, wissen Angehörige mit Migrationshintergrund nur wenig über pflegerische oder psychosoziale Unterstützungen und Leistungen im Rahmen der Palliativversorgung.

\section{KASTEN 2: AUSSAGEN AUS DEN WÖRTLICH TRANSKRIBIERTEN ANGEHÖRIGENINTERVIEWS}

Zitat 4: „sie hatte einen anaphylaktischen Schock es könnte auch viel schlimmer sein und ich weiß dass es nur passiert ist wegen der Sprache weil sie also nicht genug genug wahrgenommen wird und solche Kleinigkeiten wegen diese drei Schwerpunkten deshalb also ganz wichtig oder ich sehe das zu mindestens so dass es ganz wichtig ist dass ich immer dabei bin, zum Beispiel weil Mutter als dumm wahrgenommen wird es ist nicht genug sehr oft einfach einen Übersetzer dabei zu haben“ Frau T., Tochter, $31 \mathrm{~J}$.

Zitat 5: „wenn Untersuchungsergebnisse besprochen werden, dann ist das immer so, dass die Ärzte gar nicht sehen, dass das meine Mutter ist, die da untersucht wird, und ich nicht nur der Übersetzer bin“

Herr D., Sohn, 40J. 


\section{Versorgende: Versorgung zwischen Anspruch und Wirklichkeit}

Auch in den Versorgerinterviews werden Kommunikationsprobleme hervorgehoben. So greifen Versorger aus der Hospiz- und Palliativversorgung in der Regel spontan auf familiäre und sich zufällig ergebende Sprachmittler (etwa Reinigungskräfte oder Fahrer) zurück. Der Einsatz von medizinisch-professionellen und -geschulten Sprachmittlern ist dagegen selten, ihre Finanzierung häufig unklar; die Suche nach geeigneten Übersetzern wird als Mehraufwand von den Versorgenden eingeschätzt. Auch bestehende Strukturen werden kritisch gesehen: Versorger, die vor Ort über Sprachmittlerdienste verfügen, sehen dies als einen klaren Versorgungsvorteil. Viele Versorger sind nicht zufrieden mit den Übersetzungen und wünschen sich mehr Personal mit Migrationshintergrund oder Multiplikator (z. B. aus einer religiösen oder ethnischen Community), die zwischen den Behandelnden und den Patienten sowie ihren Angehörigen kompetent vermitteln (Kasten 3, Zitat 6).

Versorger nehmen Erwartungen der Patienten an das medizinische System in Deutschland wahr, denen sie häufig nicht entsprechen können. Mehr noch erleben sie sich in ihrer Tätigkeit als handlungsohnmächtig, besonders dann, wenn sie nicht wissen, ob sie das Thema Sterben und Tod ansprechen dürfen (Kasten 3, Zitat 7). Manchmal werden klinische Strukturen und rechtliche Vorgaben als zu starr empfunden, weil Wünsche am Lebensende (wie die Rückkehr in die Heimat) aus finanziellen und rechtlichen Gründen nicht erfüllt werden können. Zuweilen zeigen sich in den Schilderungen innere Konflikte, weil Ansprüche an eine umfassende palliativmedizinische Versorgung und die Realität der Behandlungssituation, in der die Möglichkeiten eingeschränkt scheinen, zu weit auseinanderliegen. In diesem Fall streben einige Versorger unkonventionelle Lösungen an, indem beispielsweise Rückkehrwünsche durch Spenden ermöglicht werden.

Eine migrationsspezifische geringe Inanspruchnahme des palliativen Angebots wird von vielen Versorgern und Schnittstellenakteuren in den Interviews mit einem Versorgungsanspruch durch die Familie, durch andere kulturelle Gepflogenheiten oder damit, dass bei vielen Menschen mit Migrationshintergrund das Konzept der Palliativversorgung nicht bekannt ist, begründet. Der vermeintlich kulturell fremde Hintergrund verunsichert viele Versorgende, weil sie befürchten, die Differenzen mit den gewohnten Handlungsstrategien nicht auffangen zu können. Deshalb existiert ein großes Bedürfnis nach einem interkulturellen „Leitfaden“ für einen (besseren) Umgang mit Patienten mit Migrationshintergrund (Kasten 3, Zitat 8).

Aus Sicht der Versorger beeinflussen die migrationsspezifischen Aspekte die Versorgung besonders dann, wenn sich Patienten in rechtlich unklaren und sozial unsicheren Lebenssituationen befinden. Die Ungewissheit darüber, ob medizinische Leistungen für Geflüchtete unter dem Asylbewerberleistungsgesetz gewährt und ob Familien der Patienten abgeschoben werden können, führt neben einer emotionalen zu einer zeitlichen Belastung, wenn die Versorgung von im Asylverfahren befindlichen Patienten ein Wissen um aufenthalts- und sozialrechtliche Aspekte und um Ansprechpartner zusätzlich zu den medizinisch-pflegerischen Kenntnissen verlangt.
KASTEN 3: AUSSAGEN AUS DEN WÖRTLICH TRANSKRIBIERTEN VERSORGENDENINTERVIEWS UND INTERVIEWS MIT SCHNITTSTELLENAKTEUREN

Zitat 6: „irgendwo so eine Stelle einzurichten und natürlich dann muss jemand drin sein der das fachliche Knowhow hat, der die Beziehungen hat, der weiß für den und den Patienten kann ich den und den Dolmetscher, da und da anrufen also das man sozusagen anrufen kann, hallo ich habe jetzt einen syrischen Patienten ich brauche einen Dolmetscher für diesen Patienten und dass die Wege nicht so lang sind das wirklich eine Person das koordiniert“

Frau R., Ärztin Allgemeine Palliativmedizin, 51 J.

Zitat 7: „das ist auch was ein Stück weit ja berührt belastet weil das kann man ja auch als Niederlage ein Stück weit auch für sich selber verbuchen wo man sagt ich hab ich hab den Menschen eigentlich nicht erreicht, ich hab zwar medizinisch sicherlich alles getan was dann sinnvoll und auch notwendig war aber ist es das gewesen was sich diese Patientin eigentlich gewünscht hat?“

Frau S., Ärztin Spezialisierte ambulante Palliativmedizin, $41 \mathrm{~J}$.

Zitat 8: „es gibt schon kulturelle Dinge halt die ähm einfach, spezifischer sind, das geht nicht darum dass ich, dass alles, mitmachen muss ich kann aber manchmal Verständnis dafür aufbauen sind manchmal Situationen halt die einfach blöd sind und die sind scheiße, und wenn man sie dann zwei drei Mal von einer, bestimmten Gruppe erlebt münzt man es drauf, ich kann aber trotzdem wenn ich ein Verständnis dafür habe, für die Situation, kann ich das manchmal bisschen anders einsortieren“

Herr M., Pflegekraft Palliativstation, 42 J.

\section{Diskussion}

Ein zentrales Studienergebnis ist, dass Krankheit und Migration keine kurzfristigen Brüche im biografischen Verlauf darstellen, sondern die Wahrnehmung der Patienten nachhaltig prägen [23]. Patienten mit Migrationsgeschichte, die seit vielen Jahren in Deutschland leben, nehmen im Laufe ihrer medizinischen Versorgung Abgrenzungen wahr, die sie ihrem Migrationshintergrund zurechnen. Hier hat sich der multiperspektivische Ansatz der Studie bewährt, weil er Dynamiken zwischen den Beteiligten sichtbar macht, denen Beachtung geschenkt werden sollte. Die Interviews haben gezeigt, dass die Vorstellungen der Versorgenden von kulturellen Grenzen und von Unterstützungsstrukturen in den Familien mit Migrationsgeschichte häufig ohne spezifische Kenntnisse der individuellen Biografie zur Erklärung für eigene Grenzen des Handelns herangezogen werden [24]. Auffallend in diesem Zusammenhang ist, dass viele der interviewten oder angefragten Versorgenden bei Patienten mit Migrationshintergrund sogleich an Geflüchtete und weniger an Menschen, die seit Jahren in Deutschland leben, dachten.

In der Untersuchung finden sich die auch in anderen Studien hervorgehobenen Sprachdifferenzen, die zu einer Problematisierung der Gruppe von Patienten mit Migrationshintergrund führen 
können [8]. Diese Differenzen sind jedoch kein Beleg für kulturelle Unterschiede, und die sprachlichen Barrieren müssten kein Problem sein, wenn es geeignete Sprachmittlerstrukturen gäbe. Problematisch sind verallgemeinerte Zuschreibungen, die das Verhalten der Patienten auf eine fremde Kultur zurückführen, und das Fehlen der über medizinische Belange hinausgehenden Unterstützung und Vernetzung der Versorgenden. Für eine gleichberechtigte Inanspruchnahme palliativer Versorgungsstrukturen ist die sprachliche Verständigung wichtig [25, 26]. Medizinisch ausgebildete Dolmetscher fehlen ebenso wie politisch geförderte Finanzierungsstrukturen, die eine professionelle Unterstützung gewährleisten. Durch von professionellen Sprachmittlern geleistete Übersetzungen werden Patienten medizinische Aufklärung und das Treffen selbstbestimmter behandlungsrelevanter Entscheidungen ermöglicht [27]. Patienten, ihre Angehörigen und auch die Versorger benötigen vor allem psychosoziale und politischrechtliche sowie institutionelle Unterstützung, die die migrationsspezifischen Einflüsse auf die medizinische Behandlungssituation berücksichtigen.

Die in Studien formulierte Vermutung, dass der Zugang zur gesundheitlichen Versorgung für Menschen mit Migrationshintergrund generell schwieriger ist als für Menschen ohne Migrationshintergrund, kann aufgrund der hier vorliegenden Studienergebnisse nur eingeschränkt beantwortet werden: Zumindest die Menschen mit Migrationshintergrund, die an dieser Studie teilgenommenen haben, sind in der Palliativversorgung angekommen, da sie sich bereits in Krankenhausstrukturen mit einem Palliativnetzwerk befanden.

Durch die qualitative Herangehensweise an die Erlebnisse der Patienten mit Migrationshintergrund und dadurch, dass in den Erhebungsgruppen verschiedene Perspektiven und Herkunftskontexte berücksichtigt wurden, konnte eine Vielzahl von Ausgrenzungserfahrungen oder -befürchtungen herausgearbeitet werden. Zukünftige Forschungen könnten die konkreten Auswirkungen politisch-rechtlicher Strukturen auf ungleiche Sterbesituationen verstärkt in den Blick nehmen. Vor diesem Hintergrund muss reflektiert werden, für welche Patienten das Versorgungssystem „gemacht und gedacht“ ist, und ob nicht z. B. für die Gruppe der Menschen mit Migrationshintergrund und Geflüchteten durch eine flexiblere Versorgungstruktur auf die veränderten gesellschaftlichen Bedingungen angemessener reagiert werden kann [28].

\section{Limitationen}

Die Rekrutierung der Studienteilnehmer gestaltete sich aufgrund des gesundheitlichen Allgemeinzustands der Patienten, deren zeitlich begrenzter Ressourcen und wegen der geringen Anzahl an Patienten mit Migrationshintergrund schwierig. Die Fallzahl blieb gering. Wegen des Gesundheitszustands der Patienten verzögerten sich Interviewtermine. Außerdem hat das medizinische Personal bei der Rekrutierung häufig Patienten für die Interviews vermittelt, die einen bestimmten Migrationshintergrund hatten oder aktuell geflüchtet waren. So wurde die Auswahl der Interviewpartner von den Ärzten mitbestimmt. Offen bleibt in der Studie, wie sich die Grenzen des Versorgungssystems darstellen, wenn die Gruppen mit und ohne Migrationshintergrund verglichen werden.

\section{KERNAUSSAGEN}

- Die Palliativversorgung von Menschen mit Migrationshintergrund findet häufig ohne spezifische Kenntnisse der Migrationsbiografie statt, sodass Versorgende und Patienten nicht immer eine Basis haben, auf der die Versorgung stattfinden kann. Diese Kenntnisse sind Bedingung für eine umfassende Versorgung am Lebensende.

- Medizinisch Versorgende sehen vor allem kulturelle Unterschiede als Grund für Probleme in der Versorgung am Lebensende.

- Patienten mit Migrationshintergrund formulieren Bedürfnisse, die neben medizinische auch soziale Kompetenzen der Versorgenden ansprechen, und nicht in erster Linie kulturspezifische Wünsche, wie die Versorgenden annehmen.

- Die Patienten erfahren bürokratische Hindernisse und soziale Abgrenzungen nicht nur in der Palliativversorgung, sondern oft bereits weit vor der letzten Lebensphase.

- Besonders der Statusverlust, der mit der Migration und der Krankheit einhergehen kann, wird von den Patienten als bedrohlich erlebt.

Interessenkonflikt

Die Autorinnen/Autoren geben an, dass kein Interessenkonflikt besteht.

Literatur

[1] Ther P. Die Außenseiter: Flucht, Flüchtlinge und Integration im modernen Europa. Berlin: Suhrkamp; 2018

[2] Schneider C, Mohsenpour A, Joos S et al. Health status of and health-care provision to asylum seekers in Germany: protocol for a systematic review and evidence mapping of empirical studies. Syst Rev 2014; 3: 139-146. http://systematicreviewsjournal.biomedcentral.com/articles/10.1186/ 2046-4053-3-139 (last accessed on November 2018)

[3] Spura A, Kleinke M, Robra BP et al. Wie erleben Asylsuchende den Zugang zu medizinischer Versorgung? Bundesgesundheitsblatt - Gesundheitsforschung - Gesundheitsschutz 2017; 60 (4): 462-470

[4] Brzoska P, Razum O. Gleich gesund und gut versorgt? Zur gesundheitlichen Lage und Versorgung von Menschen mit Migrationshintergrund. MDK Forum 2012 Feb; 5-6

[5] Borde T. Migrantinnen und Migranten in der deutschen Gesundheitsversorgung: Auch nach 50 Jahren der Zuwanderung noch ein Problem? In: Kollak I, Kolodziej-Durnas A, Herausgeber Formen des sozialen Lebens im multikulturellen Europa Polnische und deutsche Introspektiven. Uckerland: Schibiri-Verlag; 2008: 10-19

[6] Knipper M, Bilgin Y. Migration und Gesundheit. Sankt Augustin, Berlin: Konrad-Adenauer-Stiftung; 2009

[7] Olbermann E. Schlussbericht zum Forschungsprojekt „Gesundheitsförderung und Primärprävention bei älteren Menschen mit Migrationshintergrund“. Dortmund: Forschungsgesellschaft für Gerontologie. Institut für Gerontologie an der TU Dortmund 2011. www.gesundheitliche-chancen gleichheit.de/docext1. php?idx=276\&pfb=44518 (last accessed on November 2018)

[8] Razum O, Karrasch L, Spallek J. Migration: Eine vernachlässigte Dimension gesundheitlicher Ungleichheit? Bundesgesundheitsblatt - Gesundheitsforschung - Gesundheitsschutz 2016; 59 (2): 259-265 
[9] Razum O, Geiger I, Zeeb H et al. Gesundheitsversorgung von Migranten. Dtsch Arztebl 2004; 101 (43): 2882-2887

[10] Razum O, Zeeb H, Schenk L. Ähnliche Krankheiten, unterschiedliche Risiken. Dtsch Ärztebl 2008; 105 (47): 2520-2522

[11] Henke A, Thuss-Patience P, Behzadi A et al. End-of-life care for immigrants in Germany: an epidemiological appraisal of Berlin. Plos One 2017; 12 (8): e0182033

[12] Migala S, Bakadorova O, Flick U. Palliative Lebensqualität: Facetten der Bedürfniskommunikation russischsprachiger Migrant_innen am Lebensende. Gesundheitswesen 2015; 77: A358

[13] Henke O, Mauter D, Behzadi A et al. „Schmerzen sind eher zu ertragen als das Alleinsein“. Z Für Palliativmedizin 2015; 16 (6): 254-263

[14] Jansky M, Owusu-Boakye S, Nauck F. Palliative Versorgung von Menschen mit türkischem oder arabischem Migrationshintergrund in Niedersachsen: eine Befragung spezialisierter Palliativversorger. Bundesgesundheitsblatt Gesundheitsforschung - Gesundheitsschutz 2017; 60 (1): 45-54

[15] Paal P, Bükki J. "If I had stayed back home, I would not be alive any more...": exploring end-of-life preferences in patients with migration background. Bussmann R, Herausgeber. Plos One 2017; 12 (4): e0175314

[16] Ilkilic I. Kulturelle Aspekte bei ethischen Entscheidungen am Lebensende und interkulturelle Kompetenz. Bundesgesundheitsblatt - Gesundheitsforschung - Gesundheitsschutz 2008; 51 (8): 857-864

[17] de Graaff F, Francke A, van den Muijsenbergh M et al. „Palliative care“: a contradiction in terms? A qualitative study of cancer patients with a Turkish or Moroccan background, their relatives and care providers. BMC Palliat Care 2010; 9 (1): 19

[18] Bosma H, Apland L, Kazanjian A. Review: cultural conceptualizations of hospice palliative care: more similarities than differences. Palliat Med 2010; 24 (5): 510-522
[19] Statistisches Bundesamt: Bevölkerung und Erwerbstätigkeit: Bevölkerung mit Migrationshintergrund - Ergebnisse des Mikrozensus 2016. Statistisches Bundesamt. 2016; 1(2.2)

[20] Loch U, Rosenthal G. Das narrative Interview. In: Schaeffer D, MüllerMundt G, (eds.) Qualitative Gesundheits- und Pflegeforschung. Bern, Schweiz: Hans Huber; 2002: 221-232

[21] Strauss AL, Corbin JM. Grounded Theory: Grundlagen qualitativer Sozialforschung. Weinheim: Beltz, Psychologie VerlagsUnion; 1996

[22] Strübing J. Grounded Theory. Wiesbaden: VS Verlag für Sozialwissenschaften; 2008

[23] Schulze H. Migrieren - Arbeiten - Krankwerden: eine biografietheoretische Untersuchung. Bielefeld: Transcript-Verl; 2006: 280 (Transcript Kultur und soziale Praxis)

[24] Banse C. Komplexe Grenzziehungen und ungewisse Grenzdynamiken. Zur Palliativversorgung von Menschen mit Migrationshintergrund und Geflüchteten. Berliner Debatte Initial 2018; 29 (1): 84-94

[25] Salis Gross C, Soom Ammann E, Sariaslan E et al. Chancengleiche Palliative Care. Bedarf und Bedürfnisse der Migrationsbevölkerung in der Schweiz. München: Akademische Verlagsgemeinschaft München; 2014

[26] Owusu-Boakye S, Banse C. Narrative Interviews mit Menschen mit Migrationshintergrund im palliativen Forschungskontext. Z Für Palliativmedizin 2017; 18: 133-136

[27] Salman R. Dolmetscher im Sozial- und Gesundheitswesen - sprachliche, konzeptionelle, qualitative und politische Aspekte. NRW Landesinstitut für den öffentlichen Gesundheitsdienst (eds.): Migration und Öffentlicher Gesundheitsdienst. In: 19 Bielefeld: lögd: Wissenschaftliche Reihe; 2005: 158-172

[28] Banse C, Nauck F. Spirituelle Bedürfnisse schwer kranker Menschen mit Fluchterfahrung. Herausforderung für die Palliativversorgung. Spiritual Care [im Druck]. 